Pacific Journal of Mathematics

NEIGHBOR RELATIONS ON THE CONVEX OF CYCLIC 


\section{NEIGHBOR RELATIONS ON THE CONVEX \\ OF CYCLIC PERMUTATIONS}

\section{HELLER}

1. Introduction and summary. Two vertices of a polyhedron are called neighbors of order $k$ when they have a face of dimension $k$, and none of lower dimension, in common. $K(P)$ denotes the maximum value of $k$ for a given polyhedron $P$. For the convex hull (polyhedron) $P_{n}$ of all permutations of $n$ elements (represented by square matrices of order $n$ and interpreted as points in $n^{2}$-space) it was shown [1 and 2] that $K(P)=$ [n/2] (that is, the largest integer not exceeding $n / 2$ ), which is rather small as compared with $\operatorname{dim} P_{n}=(n-1)^{2}$. For the convex hull $Q_{n}$ of all cyclic permutations of $n$ elements that leave no element fixed, $H$. Kuhn performed computations showing that any two vertices of $Q_{5}$ but not any two vertices of $Q_{0}$ are neighbors of order 1 , which means that $K\left(Q_{5}\right)=1$ and $K\left(Q_{6}\right)>1$. The present note, dealing with general $n$, proves, for $n \geqq 8$ :

$$
\begin{aligned}
& K\left(Q_{n}\right)=K\left(P_{n}\right)-1=\frac{n}{2}-1 \text { if } n=4 m+2 \\
& K\left(Q_{n}\right)=K\left(P_{n}\right)=\left[\frac{n}{2}\right] \text { if } n \neq 4 m+2
\end{aligned}
$$

For $n=1,2, \cdots 6,7, K\left(Q_{n}\right)=0,0,1,1,1,2,2$ respectively.

2. A permutation $p$ of $n$ numbered elements is customarily represented by a matrix $\left(p_{i j}\right)$, where

$$
p_{i j}= \begin{cases}1 & \text { when } p \text { sends } i \text { into } j \\ 0 & \text { otherwise. }\end{cases}
$$

To the product of permutations then corresponds the product of the associated matrices under ordinary matrix multiplication, and therefore the same symbol will be used for a permutation and its matrix.

The following facts from [1] and [2] regarding neighbor relations on $P_{n}$ will be used in the sequal:

Received July 8, 1955. Work done under the sponsorship of the Office of Naval Research.

Reproduction in whole or in part is permitted for any purpose of the United States Government. 


$$
K\left(P_{n}\right)=\left[\frac{n}{2}\right]
$$

(2.2) $\quad p_{1}$ and $p_{2}$ are neighbors of order $k$ on $P_{n}$ if and only if $p_{1}^{-1} p_{2}$ is a product of $k$ disjoint cycles (not counting cycles of length 1)

(2.3) If $c_{1}, c_{2}, \cdots, c_{k}$ are disjoint cycles and $F$ is the face of lowest dimension that contains the two vertices

$$
p \text { and } \bar{p}=p c_{1} c_{2} \cdots c_{k},
$$

then $F$ has the $2^{k}$ vertices

$$
p c_{i_{1}} c_{i_{2}} \cdots c_{i_{s}} \quad(0 \leqq s \leqq k) .
$$

3. If the vertices of a convex polyhedron $Q$ are a subset of the vertices of a convex polyhedron $P$, let two vertices $q_{1}, q_{2}$ of $Q$ be neighbors of order $k$ on $P$ and $k^{*}$ on $Q$ :

$$
k=k\left(q_{1}, q_{2} ; P\right), \quad k^{*}=k^{*}\left(q_{1}, q_{2} ; Q\right) .
$$

Let

$$
F=F\left(q_{1}, q_{2} ; P\right), \quad F^{*}=F^{*}\left(q_{1}, q_{2} ; Q\right)
$$

be the face of lowest dimension of $P$ respectively $Q$ that contains $q_{1}$ and $q_{2}$, so that

$$
k=\operatorname{dim} A\left(F^{\top}\right), \quad k^{*}=\operatorname{dim} A\left(F^{*}\right),
$$

where $A(F)$ and $A\left(F^{*}\right)$ denote the "affine span" of $F$ and $F^{*}$ respectively, which is also obtained as the intersection of all hyperplanes that support $P$ respectively $Q$ and contain $q_{1}$ and $q_{2}$ (with the understanding that $A$ is the entire space when such hyperplanes do not exist); then

$$
F \supseteqq F^{*},
$$

hence

$$
A\left(F^{\prime}\right) \supseteqq A\left(F^{*}\right),
$$

and therefore

$$
k \geq k^{*}
$$

Proof of (3.1). The line segment joining $q_{1}$ and $q_{2}$ goes through the interior of $F^{*}$ (otherwise $q_{1}$ and $q_{2}$ would have a face of lower dimension in common). Therefore any hyperplane through $q_{1}$ and $q_{2}$ necessarily contains interior points of $F^{*}$. 
Further, the vertices of $Q$, hence in particular those of $F^{*}$, are also vertices of $P$. Therefore any hyperplane that supports $P$ supports $F^{* *}$.

Above establishes that any hyperplane $H$ that supports $P$ and contains $q_{1}$ and $q_{2}$ necessarily contains $F^{*}$, since it supports $F^{*}$ and contains points interior to $F^{*}$. Therefore

$$
A\left(F^{\prime}\right) \supseteqq F^{*},
$$

which, in conjunction with

$$
P \supset Q \supset F^{*}
$$

implies

$$
F^{*} \leqq P \cap A(F) .
$$

This completes the proof of (3.1), since the right hand side of the last relation equals $F$.

A somewhat sharper form of (3.1) may be noted as

LEMma 1. The vertices of $F^{*}$ are among the vertices of $F$.

The proof is immediate from (3.1) and the fact that the vertices of $F^{*}$ are vertices of $P$, and a vertex of $P$ contained in $F$ is vertex of $F$.

From (3.3) it follows that $\max k^{*} \leqq \max k$, that is

$$
K(Q) \leqq K(P)
$$

4. At this point it is convenient to first establish some auxiliary facts. $p, q, c$ denote permutations of $n$ elements, for fixed $n$.

LEMMA 2. If

$$
c_{1}, c_{2}, \cdots, c_{r}, c_{r+1}, \cdots, c_{s}
$$

is a set of $s$ disjoint cycles, and

$$
c^{\prime}=c_{1} c_{2} \cdots c_{r}, \quad c^{\prime \prime}=c_{r+1} c_{r+2} \cdots c_{s}
$$

then

$$
c^{\prime}+c^{\prime \prime}=I+c^{\prime} c^{\prime \prime}
$$

Proof. Obvious (note that a cycle of less than $n$ elements is still represented as an $n$ by $n$ matrix, with 1 's along the main diagonal for fixed elements). 
LEMma 3. Under the assumptions of Lemma 1, let

$$
q, q c^{\prime}, q c^{\prime \prime}, q c^{\prime} c^{\prime \prime}=\bar{q}
$$

be vertices of a polyhedron $R$. Then

a hyperplane $H$ through $q$ and $\bar{q}$ that supports $R$ contains $q c^{\prime}$ and $q c^{\prime \prime}$,

and consequently

$F(q, \bar{q} ; R)$ contains $q c^{\prime}$ and $q c^{\prime \prime}$ (obviously as vertices).

This lemma will be used in the particular case where $R=Q_{n}$ or $P_{n}$.

Proof of Lemma 3. Using parentheses to denote the inner product, let $H$, given by $(h, x)=\alpha$, contain $q$ and $\bar{q}$ but not contain $q c^{\prime}$ (say); that is

$$
(h, q)=(h, \bar{q})=\alpha, \quad\left(h, q c^{\prime}\right)=\alpha+\beta, \quad \beta \neq 0 .
$$

By (4.1) and (4.2)

$$
q c^{\prime}+q c^{\prime \prime}=q+\bar{q},
$$

hence

$$
\left(h, q c^{\prime \prime}\right)=\left(h, q+\bar{q}-q c^{\prime}\right)=2 \alpha-(\alpha+\beta)=\alpha-\beta,
$$

so that $H$ separates $q c^{\prime}$ from $q c^{\prime \prime}$ and therefore does not support $R$.

LEMMA 4. If

$$
\begin{aligned}
& k=\left[\begin{array}{l}
n \\
2
\end{array}\right], \quad 2 s \leqq k \\
& q=(12 \cdots n) \\
& c_{i}=(i, i+k) \quad(i=1,2, \cdots k),
\end{aligned}
$$

then the product of $q$ with $2 s$ distinct $c_{i}$,

$$
q c_{i_{1}} c_{i_{2}} \cdots c_{i_{2}}
$$

is an $n$-cycle.

Proof. Since the $c_{i}$ are disjoint, they commute, and may be arranged in such manner that

$$
i_{1}<i_{2}<\cdots<i_{2 s}
$$


then

$$
\begin{aligned}
& (1 \cdots n)\left(i_{1}, i_{1}+k\right)\left(i_{2}, i_{2}+k\right) \cdots\left(i_{2 s-1}, i_{2 s-1}+k\right)\left(i_{2 s}, i_{2 s}+k\right) \\
= & \left(1 \cdots i_{1}, i_{1}+k+1, \cdots i_{2}+k, i_{2}+1, \cdots i_{3}, i_{3}+k+1, \cdots i_{4}+k, i_{4}+1 \cdots\right. \\
& \cdots i_{2 s-1}, i_{2 s-1}+k+1, \cdots i_{2 s}+k, i_{2 s}+1, \cdots \\
& i_{1}+k, i_{1}+1, \cdots i_{2}, i_{2}+k+1, \cdots i_{3}+k, i_{3}+1, \cdots i_{4}, i_{1}+k+1, \cdots \\
& \left.\cdots i_{2 s-1}+k, i_{2 s-1}+1, \cdots i_{2 s}, i_{2 s}+k+1, \cdots n\right) .
\end{aligned}
$$

It is easily verified above relation also holds, with proper changes, for $i_{1}=1$ and for $2 s=k, 2 k=n$.

In similar straightforward fashion one easily proves:

LemMA 5. If $q$ is an n-cycle and $d$ is a 3-cycle, then $q d$ is an ncycle if and only if the elements of $d$ occur in $q$ in the same cyclic order as in $d$.

Lemma 6. If $q$ is an n-cycle and the 2-cycle $(i j) \neq(\mathrm{km})$, then $q(i j)(\mathrm{km})$ is an $n$-cycle if and only if the pair $i, j$ separates the pair $k$, $m$ in $q$.

5. The case $n=4 m, n=4 m+1 ; m \geqq 2$.

$$
K\left(Q_{n}\right)=K\left(P_{n}\right) \quad(n=4 m, 4 m+1 ; m \geqq 2)
$$

Proof. Because of (3.4), it is sufficient to show that $K\left(Q_{n}\right) \geqq K\left(P_{n}\right)$; this will be achieved by showing that for a particular pair of vertices $q, \bar{q}$

$$
k\left(q, \bar{q} ; Q_{n}\right) \geqq\left[\frac{n}{2}\right]=K\left(P_{n}\right)
$$

Now let $2 m=k$, so that $n \geqq 2 k$, choose

$$
\left\{\begin{array}{l}
q=(12 \cdots n) \\
c_{s}=(i, i+k) \quad(i=1,2 \cdots k) \\
\bar{q}=q c_{1} c_{2} \cdots c_{k}=q c,
\end{array}\right.
$$

and denote by $c^{\prime}$ the product of an even number (including 0 and $k$ ) of the $c_{i}$, by $c^{\prime \prime}$ the product of the remaining $c_{i}$ (whose number is also even, since $k$ is even):

$$
\left\{\begin{array}{c}
c^{\prime}=c_{i_{1}} c_{i_{2}} \cdots c_{i_{2 s}} \quad(0 \leqq 2 s \leqq k) \\
c^{\prime} c^{\prime \prime}=c_{1} c_{2} \cdots c_{k}=c .
\end{array}\right.
$$


(It should be noted that the now following proof of $k^{*}\left(q, \bar{q} ; Q_{n}\right) \geqq$ $k$ does not depend on the special assumption $n=4 m, 4 m+1$ and $k=2 m$, but rather holds in general for any pair $n, k$, where $k$ is even and $n \geq$ $2 k$; this fact will be used in $\S 9$ ).

The $q c^{\prime}$ are vertices of $Q_{n}$ (by Lemma 4) and therefore (by Lemma 3) they are also vertices of $F^{*}=F\left(q, \bar{q} ; Q_{n}\right)$.

To verify (5.2), that is

$$
\operatorname{dim} A\left(F^{*}\right) \geq k,
$$

consider the following subset of $k+1$ vertices of $F^{*}$ :

$$
q_{1}=q c_{1} c_{1}=q, q_{2}=q c_{1} c_{2}, \cdots q_{k}=q c_{1} c_{k}, q_{k+1}=q c=\bar{q} .
$$

The $q_{i}$ of (5.5) are linearly independent.

Proof. Assume

$$
\lambda q c+\sum_{i=1}^{k} \lambda_{i} q_{i}=0 .
$$

Successive application of (4.1) to

$$
c=c_{1} c_{2} \cdots c_{k}
$$

yields

$$
c=c_{1}\left[c_{2}+\cdots+c_{k}-(k-2) I\right],
$$

and (5.6) becomes

$$
\lambda q c_{1}\left[c_{2}+\cdots+c_{k}-(k-2) I\right]+\sum_{i=1}^{k} \lambda_{i} q c_{1} c_{i}=0
$$

that is

$$
q c_{1}\left[\lambda_{1} c_{1}-\lambda(k-2) I+\sum_{i=2}^{k}\left(\lambda_{i}+\lambda\right) c_{i}\right]=0
$$

or, equivalently, since $q$ and $c_{1}$ are nonsingular matrices

$$
\lambda_{1} c_{1}-\lambda(k-2) I+\sum_{i=2}^{k}\left(\lambda_{i}+\lambda\right) c_{i}=0
$$

Since the $c_{i}$ are disjoint cycles (5.8) implies

$$
\lambda_{1}=0 ; \lambda_{i}+\lambda=0(i=2, \cdots k) ; \lambda(k-2)=0
$$

which, in conjunction with $k \neq 2$ (following from $m \geqq 2$ ), further implies

$$
\lambda=0, \quad \lambda_{i}=0 .
$$


This verifies that the $k+1 q_{i}$ of (5.5) are linearly independent, so that the dimension of their linear span is $k+1$, and therefore the dimension of their affine span equal to $k$. This completes the proof of (5.2) and hence of $(5.1)$

6. The case $n=4 m, n=4 m+1 ; m=1$. Removing the restriction $m$ $\geqq 2$ in (5.1) leaves the cases $n=4$ and $n=5$ still to be considered

$$
K\left(Q_{n}\right)=1 \quad(n=4,5)
$$

Proof. Since, by (3.4) and (2.1), $K\left(Q_{n}\right) \leqq 2$, one only has to show that $K\left(Q_{n}\right) \neq 2$.

Assume there were two vertices $q$ and $\bar{q}$ of $Q_{n}$ such that

$$
k^{*}\left(q, \tilde{q} ; Q_{n}\right)=2 \text {. }
$$

Then, by (3.4), (3.3) and (2.1)

$$
k\left(q, \bar{q} ; P_{n}\right)=2,
$$

which by (2.2) implies that $q^{-1} \bar{q}$ is a product of two disjoint cycles, say $c_{1}, c_{2}$, so that $\bar{q}=q c_{1} c_{2}$.

Since $q$ and $\bar{q}$ are cycles of the same length (namely $n$ ), $c_{1} c_{2}$ is necessarily an even permutation, so that $c_{1}$ and $c_{2}$ are both of length 2 .

Now let $F$ be the lowest dimensional face of $P_{n}$ containing $q$ and $\bar{q}$. Then, by (2.3), $F$ has the 4 vertices

$$
q, \bar{q}, q c_{1}, q c_{2} .
$$

of which the last two are not $n$-cycles and therefore not vertices of $F^{*}$. Hence, by Lemma $1, F^{*}$ has only the two vertices $q$ and $q$, which implies $k^{*}=1$ in contradiction to the assumption that $k^{*}=2$. This completes the proof of (6.1).

7. The case $n=4 m+3 ; m \neq 1$.

$$
K\left(Q_{n}\right)=K\left(P_{n}\right) \quad(n=4 m+3, m \neq 1),
$$

including $m=0$.

Proof. Because of (3.4) it is again sufficient to point out two vertices, $q, \bar{q}$, of $Q_{n}$, such that

$$
k^{*}\left(q, \bar{q} ; Q_{n}\right) \geqq K\left(P_{n}\right)=2 m+1 .
$$

For $k=2 m$, let $q, c_{i}, c, c^{\prime}, c^{\prime \prime}$ be defined as in (5.3) and (5.4), let $d=(2 k+1,2 k+2,2 k+3)$, and $\bar{q}=q c d$. 
By Lemmas 4 and 5 the $q c^{\prime}$ and $q c^{\prime} d$ are vertices of $Q_{n}$ for all $c^{\prime}$ of (5.4), and by Lemma 3 they are also vertices of $F^{*}\left(q, \bar{q} ; Q_{n}\right)$. To prove that

$$
\operatorname{dim} A\left(F^{*}\right) \geqq 2 m+1,
$$

it is shown that the dimension of the linear span of $F^{*}$ is $\geq 2 m+2=$ $k+2$, in verifying that the $k+2$ vertices of $F^{*}$

$$
q_{1}=q=q c_{1} c_{1}, q_{2}=q c_{1} c_{2}, \cdots, q_{k}=q c_{1} c_{k}, q_{k+1}=q d, \quad q_{k+2}=\bar{q}=q c d
$$

are linearly independent.

Assume

$$
\sum_{i=1}^{k+2} \lambda_{i} q_{i}=0
$$

or, equivalently, substituting for $q_{i}$ their expressions from (7.3), omitting the non singular common factor $q c_{1}$, and writing $\mu_{i}$ for $\lambda_{k+i}$,

$$
\sum_{i=1}^{k} \lambda_{i} c_{i}+\mu_{1} c_{1} d+\mu_{2} c_{2} c_{3} \cdots c_{k} d=0 .
$$

Application of (4.1) yields for the left hand side of (7.5)

$$
\sum_{i=1}^{k} \lambda_{i} c_{i}+\mu_{1}\left(c_{1}+d-I\right)+\mu_{2}\left[c_{2}+\cdots+c_{k}+d-(k-1) I\right],
$$

so that (7.4) is equivalent to

$$
\left(\lambda_{1}+\mu_{1}\right) c_{1}+\sum_{i=2}^{k}\left(\lambda_{i}+\mu_{2}\right) c_{i}+\left(\mu_{1}+\mu_{2}\right) d-\left[\mu_{1}+(k-1) \mu_{2}\right] I=0
$$

Since the $c_{i}$ and $d$ are disjoint cycles, (7.6) implies

$$
\left\{\begin{array}{l}
\lambda_{1}+\mu_{1}=0 \\
\lambda_{i}+\mu_{2}=0 \\
\mu_{1}+\mu_{2}=0 \\
\mu_{1}+(k-1) \mu_{2}=0
\end{array} \quad(i=2,3, \cdots k)\right.
$$

The last two relations of (7.7) imply (because of the assumption $m$ $\neq 1$, hence $k \neq 2, k-1 \neq 1$ )

$$
\mu_{1}=\mu_{2}=0,
$$

which in conjunction with the first two relations of (7.7) implies

$$
\lambda_{i}=0 \quad(i=1,2, \cdots k),
$$

so that all coefficients of (7.4) vanish; this proves that the $q_{i}$ of (7.4) 
are linearly independent, and completes the proof of (7.2) and hence (7.1).

8. The case $n=7$ (excepted in $\S 7$ ).

$$
K\left(Q_{7}\right)=K\left(P_{7}\right)-1=2
$$

Proof. By (3.4) and (2.1)

$$
K\left(Q_{7}\right) \leqq 3 .
$$

To see that equality cannot hold, let $q=(12 \cdots 7)$.

Because of (2.1) and (3.3), only such $\bar{q}$ must be considered where

$$
k\left(q, \bar{q} ; P_{\tau}\right)=3 \text {. }
$$

By (2.2) the last relation is only possible for

$$
\bar{q}=q c_{1} c_{2} d,
$$

where $c_{1}, c_{2}, d$ are disjoing cycles.

For $\bar{q}$ to be a 7-cycle it is necessary (not sufficient) that $c_{1} c_{2} d$ be even, that is, that two of them, say $c_{1}$ and $c_{2}$, be transpositions and $d$ a 3 cycle.

For the same reason, among the 8 vertices of $F\left(q, \bar{q} ; P_{7}\right)$ determined by (2.3), at most 4 are 7-cycles, namely

$$
q_{1}=q, q_{2}=q c_{1} c_{2}, q_{3}=q d, q_{1}=\bar{q}=q c_{1} c_{2} d,
$$

so that, by Lemma $1, F^{*}\left(q, \bar{q} ; Q_{7}\right)$ has at most the 4 vertices (8.2).

However, application of (4.1) yields

$$
q_{1}+q_{4}=q\left(I+c_{1} c_{2} d\right)=q\left(I+c_{1} c_{2}+d-I\right)=q_{2}+q_{3}
$$

which is a relation

$$
\sum \lambda_{i} c_{i}=0 \quad \text { with } \quad \Sigma \lambda_{i}=0,
$$

therefore

$$
\operatorname{dim} A\left(F^{*}\right) \leqq 2 .
$$

It has thus been established that

$$
K\left(Q_{i}\right) \leqq 2 .
$$

To complete the proof of (8.1), choose

$$
q=(12 \cdots 7), c_{1}=(13), c_{2}=(24), d=(567) .
$$

Then each $q_{i}$ of $(8,2)$ is a 7-cycle (by Lemmas 4 and 5) and a 
vertex of $F^{*}\left(q, \bar{q} ; Q_{7}\right)$ (by Lemma 3.) The last 3 of these $q_{i}$ are linearly independent. This establishes, for this particular face $F^{*}$,

$$
\operatorname{dim} A\left(F^{*}\right)=2,
$$

and completes the proof of (8.1).

9. The case $n=4 m+2$.

$$
K\left(Q_{n}\right)=K\left(P_{n}\right)-1=2 m \quad(n=4 m+2) .
$$

The proof is achieved in showing

$$
\begin{aligned}
& K\left(Q_{n}\right) \leqq K\left(P_{n}\right)-1=2 m \\
& K\left(Q_{n}\right) \geqq K\left(P_{n}\right)-1=2 m .
\end{aligned}
$$

To verify (9.2), assume $K\left(Q_{n}\right)>K\left(P_{n}\right)-1$, which, by (3.4) and (2.1), implies $K\left(Q_{n}\right)=K\left(P_{n}\right)=2 m+1$.

Then there must be a pair of vertices $q$ and $\bar{q}$ on $Q_{n}$ such that

$$
k^{*}\left(q, \bar{q} ; Q_{n}\right)=2 m+1,
$$

and hence, by (3.3) and (2.1),

$$
k\left(q, \bar{q} ; P_{n}\right)=2 m+1,
$$

which, by (2.2) implies

$$
\bar{q}=q c_{1} c_{2} \cdots c_{2 m+1},
$$

where the $c_{i}$ are disjoint cycles, and therefore necessarily transpositions, because of $n=2(2 m+1)$. Then however, the product of the $c_{i}$ is an odd permutation, and $\bar{q}$ cannot be an $n$-cycle if $q$ is one. This proves (9.2).

To verify (9.3), consider first the case $m \geq 2$. Setting $2 m=k$, the construction from (5.3) through the end of $\S 5$ proves the existence of $q, \vec{q}$ with $k^{*}\left(q, \bar{q} ; Q_{n}\right)=k$, which implies $K\left(Q_{n}\right) \geq k$.

For $m=1$, that is, $n=6$, choose

$$
q=(12 \cdots 6), d_{1}=(123), d_{2}=(456), \bar{q}=q d_{1} d_{2} .
$$

Then, by Lemma 5 , the 4 points

$$
q, q d_{1}, q d_{2}, \bar{q}=q d_{1} d_{2}
$$

are 6-cycles, and therefore, by Lemma 3, vertices of

$$
F^{*}\left(q, \bar{q} ; Q_{6}\right) \text {. }
$$

This implies $\operatorname{dim} A\left(F^{*}\right) \geq 2$ (since not more than two vertices can be on 
a line), that is,

$$
k^{*}\left(q, \bar{q} ; Q_{6}\right) \geqq 2 .
$$

Finally (if one wants to split hairs) for $m=0$, that is, $n=2,(9.3)$ amounts to asserting the existence of at least one 2-cycle; for $q=\bar{q}=$ (12), $F^{*}\left(q, \bar{q} ; Q_{2}\right)=q, k^{*}=0$, hence $K\left(Q_{2}\right) \geqq 0$. This completes the proof of (9.1).

The relations (5.1), (6.1), (7.1), (8.1), and (9.1) constitute the statement at the end of $\S 1$.

\section{REFERENCES}

1. I. Heller, Geometric characterization of cyclic permutations, (Abstract), Bull. Amer. Math. Soc., 1955.

2. On the travelling salesman's problem, Proc. Second symposium on Linear Programming, Washington, D.C., January 29, 1955.

3. T. S. Motzkin, The assignment problem, Proc. 6th Appl. Math. Symposium, McGrawHill, 1955.

The George WAShington UnIVERSITY 



\section{PACIFIC JOURNAL OF MATHEMATICS}

\section{EDITORS}

\author{
H. L. Royden \\ Stanford University \\ Stanford, California \\ E. Hewitt \\ University of Washington \\ Seattle 5 , Washington
}

R. P. Dilworth

California Institute of Technology

Pasadena 4, California

\author{
A. HorN* \\ University of California \\ Los Angeles 24, California
}

\section{ASSOCIATE EDITORS}

\author{
E. F. BECKENBACH \\ C. E. BURGESS \\ H. BUSEMANN \\ H. FEDERER
}

\author{
M. HALL \\ P. R. HALMOS \\ V. GANAPATHY IYER \\ R. D. JAMES
}

\author{
M. S. KNEBELMAN \\ I. NIVEN \\ T. G. OSTROM \\ M. M. SCHIFFER
}

J. J. STOKER

G. SZEKERES

F. WOLF

K. YOSIDA

\section{SPONSORS}

\author{
UNIVERSITY OF BRITISH COLUMBIA \\ CALIFORNIA INSTITUTE OF TECHNOLOGY \\ UNIVERSITY OF CALIFORNIA, BERKELEY \\ UNIVERSITY OF CALIFORNIA, DAVIS \\ UNIVERSITY OF CALIFORNIA, LOS ANGELES \\ UNIVERSITY OF CALIFORNIA, SANTA BARBARA \\ MONTANA STATE UNIVERSITY \\ UNIVERSITY OF NEVADA \\ OREGON STATE COLLEGE \\ UNIVERSITY OF OREGON \\ UNIVERSITY OF SOUTHERN CALIFORNIA
}

STANFORD RESEARCH INSTITUTE

STANFORD UNIVERSITY

UNIVERSITY OF UTAH

WASHINGTON STATE COLLEGE

UNIVERSITY OF WASHINGTON

AMERICAN MATHEMATICAL SOCIETY HUGHES AIRCRAFT COMPANY SHELL DEVELOPMENT COMPANY

Mathematical papers intended for publication in the Pacific Journal of Mathematics should be typewritten (double spaced), and the author should keep a complete copy. Manuscripts may be sent to any of the editors. Manuscripts intended for the outgoing editors should be sent to their successors. All other communications to the editors should be addressed to the managing editor, Alfred Horn at the University of California, Los Angeles 24, California.

50 reprints of each article are furnished free of charge; additional copies may be obtained at cost in multiples of 50 .

The Pacific Journal of Mathematics is published quarterly, in March, June, September, and December. The price per volume (4 numbers) is $\$ 12.00$; single issues, $\$ 3.50$. Back numbers are available. Special price to individual faculty members of supporting institutions and to individual members of the American Mathematical Society: $\$ 4.00$ per volume; single issues, $\$ 1.25$.

Subscriptions, orders for back numbers, and changes of address should be sent to Pacific Journal of Mathematics, c/o University of California Press, Berkeley 4, California.

Printed at Kokusai Bunken Insatsusha (International Academic Printing Co., Ltd.), No. 10, 1-chome, Fujimi-cho, Chiyoda-ku, Tokyo, Japan.

* During the absence of E. G. Straus.

PUBLISHED BY PACIFIC JOURNAL OF MATHEMATICS, A NON-PROFIT CORPORATION COPYRIGHT 1956 BY PACIFIC JOURNAL OF MATHEMATICS 


\section{Pacific Journal of Mathematics}

\section{Vol. 6, No. $3 \quad$ BadMonth, 1956}

Richard Arens and James Eells, Jr., On embedding uniform and topological spaces ............................................ 397

N. Aronszajn and Prom Panitchpakdi, Extension of uniformly continuous transformations and hyperconvex metric spaces .............. 405

Kai Lai Chung and Cyrus Derman, Non-recurrent random walks ........ 441

Harry Herbert Corson, III, On some special systems of equations . . . . . . . . . 449

Charles W. Curtis, On Lie algebras of algebraic linear transformations . . . 453

Isidore Heller, Neighbor relations on the convex of cyclic permutations . . . . 467

Solomon Leader, Convergence topologies for measures and the existence of transition probabilities..................................... 479

D. H. Lehmer, On certain character matrices ...................... 491

Michael Bahir Maschler, Minimal domains and their Bergman kernel function ........................................... 501

Wm. M. Myers, Functionals associated with a continuous transformation ................................... 517

Irving Reiner and Jonathan Dean Swift, Congruence subgroups of matrix groups ....................................... 529

Andrew Sobczyk, Simple families of lines ................... 541

Charles Standish, A class of measure preserving transformations ........ 553 Jeremiah Milton Stark, On distortion in pseudo-conformal mapping ..... 565 